\title{
A correlation between SN II metal line strength and host $H$ II region oxygen abundance
}

\author{
Joseph P. Anderson ${ }^{1}$, Claudia P. Gutiérrez ${ }^{1,2,3}$ and Luc Dessart ${ }^{4}$ \\ ${ }^{1}$ European Southern Observatory, Alonso de Crdova 3107, Casilla 19001, Santiago, Chile \\ email: janderso@eso.org \\ ${ }^{2}$ Millennium Institute of Astrophysics, Casilla 36-D, Santiago, Chile \\ ${ }^{3}$ Departamento de Astronomía, Universidad de Chile, Camino El Observatorio 1515, Las \\ Condes, Santiago, Chile \\ ${ }^{4}$ Laboratoire Lagrange, UMR7293, Universite Nice Sophia-Antipolis, CNRS, Observatoire de \\ la Cote d'Azur, F-06300 Nice, Franc
}

\begin{abstract}
Dessart et al., demonstrated that type II supernova (SN II) model spectra present increasing metal line strength with increasing progenitor metallicity. To confront these models with observations, we obtained a large sample of SN II host H II region emission line spectroscopy. We show that inferred SN II host H II region metallicities have a statistically significant correlation with the strength of SN II metal lines, specifically Fe II $5018 \AA$.
\end{abstract}

Keywords. (stars:) supernovae: general, ISM: abundances

Knowledge of the chemical evolution of galaxies is important for our overall understanding of the Universe. Outside of our local group of galaxies the dominant source of abundance/metallicity measurements comes from emission line diagnostics. However, there are significant issues with these methods, both on the absolute abundance scale, and also of large systematic offsets between different diagnostics (see e.g. López-Sánchez et al. 2012). Hence, any independent metallicity indicator is of significant value.

Dessart et al. (2014) (following the earlier work of Dessart et al. 2013) studied the spectral properties of SN II ejecta models that resulted from the explosion of RSG stars evolved from the main sequence at different metallicities, from sub-solar to supersolar. They found that metal line strengths correlated with progenitor metallicity. As an observational follow-up of that work, we obtained host H II region spectroscopy of a large sample of SNe II. Using these data, emission line fluxes were extracted and host H II region oxygen abundances were determined using the Marino et al. (2013) O3N2 diagnostic. For each associated SN II we measured pseudo equivalent widths (pEWs) of the FeII $5018 \AA$ absorption line in the SN spectrum interpolated to 50 days post explosion.

In Fig. 1a we present a correlation between environment oxygen abundance (a proxy for metallicity) and SN Fe II $5018 \AA$ pEWs interpolated to 50 days post explosion. A statistically significant correlation is found in that SNe with higher pEWs are found to explode within higher abundance environments. This initial correlation was achieved with all SNe II irrespective of their light-curve morphology. As shown in Anderson et al. (2014) SNe II show a large range in light-curve morphologies (brightness, decline rates, optically thick phase durations etc). Variations in progenitor or explosion properties, independent of composition, could produce comparable effects to changes in metallicity. In Fig. $1 b$ we remove all SNe which we do not consider 'normal' SNe IIP. Within this 

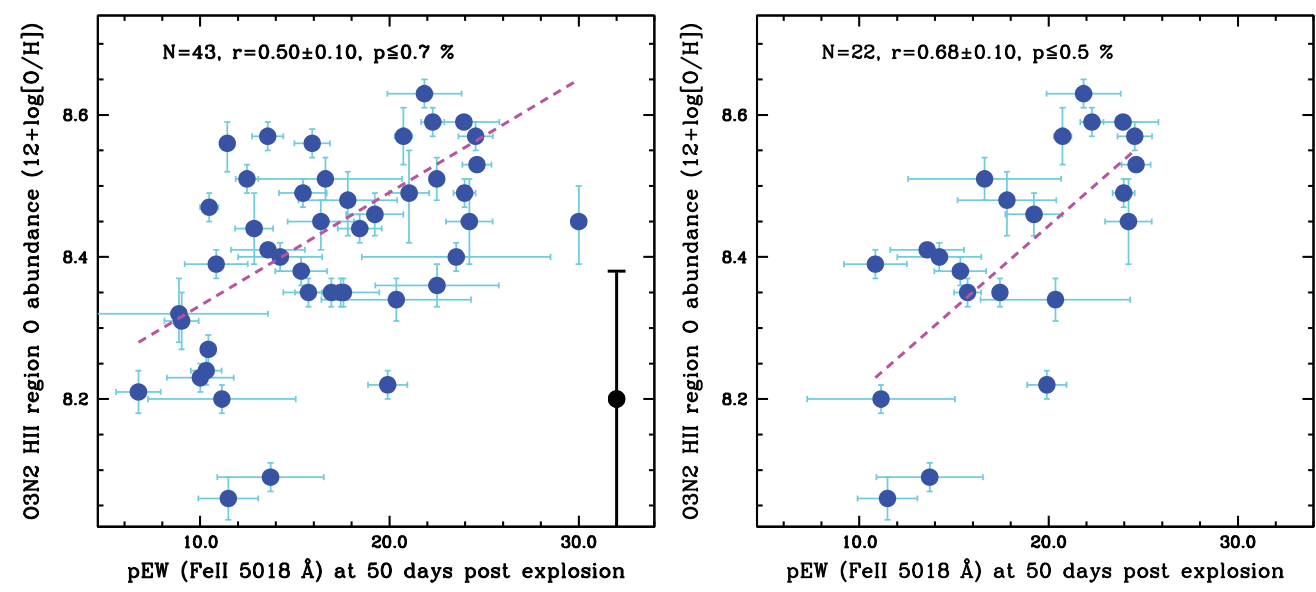

Figure 1. Left, a): SN II pEWs of Fe II $5018 \AA$ at 50 days post explosion against host H II region oxygen abundance. Right, b): same as a) but now for a subset of SNe II whih have light-curve morphologies consistent with 'normal' SNe IIP.

sub-sample the dispersion is significantly reduced. The dispersion is also reduced when we use a colour in place of time epoch.

This work strengthens the conclusions of Dessart et al. (2014), and motivates further study to enable SNe II to be used as independent metallicity indicators throughout the Universe. A full analysis will soon be published in Anderson et al., (in preparation).

\section{Acknowledgement}

we acknowledge the use of 'CATS et al.' and Carnegie Supernova Project spectroscopy (data to be published in Gutiérriez et al. in preparation).

\section{References}

Anderson, J. P., et al., 2014 ApJ, 786, 67

Dessart, L., Hillier, D. J., Waldman, R., \& Livne, E., 2013 MNRAS, 433, 1745

Dessart, L., et al., 2014 MNRAS, 440, 1856

López-Sánchez, A., et al. 2012 MNRAS, 526, 2630

Marino, R. A., et al. 2013 AESA, 559, 114 